\title{
Histórico de transtornos disfóricos no período reprodutivo e a associação com sintomas sugestivos de depressão na pós-menopausa
}

\section{Dysphoric disorders history in reproductive period and the association with symptoms suggestive of postmenopausal depression}

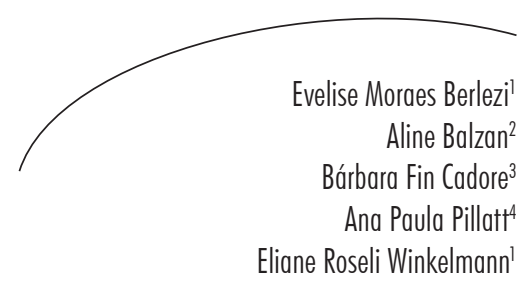

\section{Resumo}

Este estudo buscou verificar se existe associação entre o histórico de transtornos disfóricos no período reprodutivo e a presença de sintomas sugestivos de depressão na pós-menopausa. Trata-se de uma pesquisa do tipo transversal analítica. A população foram mulheres pós-menopausa, com idade entre 50 e 65 anos, com no mínimo 12 meses de amenorreia, residentes em um município do interior do Estado do Rio Grande do Sul. Atenderam aos critérios do estudo 112 mulheres. Os dados foram obtidos por meio de entrevista estruturada contendo informações sociodemográficas, anamnese para identificação da presença de transtornos disfóricos, sintomas relacionados a menopausa, tratamento de reposição hormonal e aplicada a escala de depressão de Hamilton para avaliar a presença de sintomas sugestivos de depressão. Os transtornos disfóricos mais relatados pelas mulheres foram o cansaço, as dores articulares ou musculares, cefaleia, nervosismo e irritabilidade. A maioria apresentava algum grau de sintoma depressivo e verificou-se associação entre esses sintomas e a presença de transtornos disfóricos. Embora não se tenha observado relação entre terapia de reposição hormonal e sintomas depressivos, expressivo número de mulheres apresentou este sintoma com o uso ou não de terapia de reposição hormonal. O estudo mostrou associação de transtornos disfóricos pré-menstruais e sintomas sugestivos de depressão em mulheres pós-menopausa.

\section{Abstract}

This study aimed to investigate the association between the history of dysphoric disorders in the reproductive period and the presence of symptoms suggestive of depression in postmenopausal women. Cross-sectional and analytic research whose

\footnotetext{
Departamento de Ciências da Vida. Universidade Regional do Noroeste do Estado do Rio Grande do Sul. Ijuí, RS, Brasil.

2 Serviço de Fisioterapia. Hospital Santa Lúcia. Cruz Alta, RS, Brasil.

3 Curso de Fisioterapia. Universidade Regional do Noroeste do Estado do Rio Grande do Sul. Ijuí, RS, Brasil.

4 Programa de Pós-graduação em Envelhecimento Humano. Universidade de Passo Fundo. Passo Fundo, RS, Brasil.
}

Palavras-chave: Mulheres. Menopausa. Depressão. Sintomas. 
population were post-menopausal women, aged 50 to 65 years, at least 12 months of amenorrhea, residents in a interior city from the state of Rio Grande do Sul, Brazil. Attended the criteria of the study 122 women. Data were obtained by structured interview with social demographic information, anamnesis to identify presence of dysphoric disorders, symptoms related to menopause, hormonal replacement therapy and applied the Hamilton Depression Scale to validate presence of suggestive depression symptoms. The more frequently dysphoric disorders related by women were tiredness, joint or muscular pain, headache, nervousness and irritability. Major women presented some degree of depression symptoms and it was found association between suggestive depression symptoms and dysphoric disorders. Although not observed relation between HRT and depression symptoms, expressive number of women presented this symptom using or not HRT. The study showed association between pre-mensturaldysphoric disorders and suggestive depression symptoms in post-menopausal women.
Key words: Women. Menopause. Depression. Symptoms.

\section{INTRODUÇÃO}

O aumento da expectativa de vida das mulheres gerou novos desafios na área da saúde, merecendo atenção diferenciada no período pósreprodutivo. No início do século XX, pouca atenção foi dada às mulheres nessa faixa etária, em parte, devido a menor expectativa de vida feminina até então, que não permitia à maioria das mulheres viver o suficiente para atingir o climatério. No entanto, o aumento da expectativa de vida feminina, a partir da segunda metade do século XX, mudou essa situação, desencadeando um interesse crescente pelas questões relacionadas ao envelhecimento feminino. ${ }^{1}$

Vistos antigamente como um sinal iminente do envelhecimento, o climatério e a menopausa são considerados hoje um fenômeno dos "anos intermediários". As mulheres brasileiras atingem a menopausa em média aos 51,2 anos, fato que as coloca na perspectiva de viverem muitos anos de suas vidas em estado de deficiência hormonal. ${ }^{2}$ Segundo Vieira \& Navarro, ${ }^{3}$ essa condição se associa a um hipoestrogenismo crescente, em que a deficiência de produção desse hormônio acarreta uma série de mudanças de ordem física e psicológica. Dentre as condições psicológicas, as alterações de humor podem propiciar o surgimento de episódios depressivos e influenciar na incidência de depressão nesta fase da vida da mulher.
A depressão é conceituada como um subtipo de transtorno afetivo que pode ser único ou recorrente, apresentando sintomas psíquicos (humor depressivo, fadiga, diminuição da capacidade de pensar, de tomar decisões), fisiológicos (alterações do sono, do apetite, do interesse sexual) e comportamentais (retraimento social). É de causa multifatorial, afeta mais mulheres do que homens com maior prevalência no período pós-reprodutivo. Fatores hormonais, socioculturais e comorbidades podem agir em separado ou sinergicamente como desencadeadores de sintomas depressivos, principalmente na pós-menopausa. ${ }^{4,5}$

Com relação ao fator hormonal, estudos como o de Rubinow \& Schmidt ${ }^{6}$ evidenciaram que os hormônios sexuais, especialmente o estrogênio, desempenham importante papel modulatório sobre os sistemas de neurotransmissão (dopamina, serotonina, noradrenalina, acetilcolina, do sistema GABA e glutamatérgico). Esses hormônios teriam ainda ação direta sobre mecanismos reguladores de neuroplasticidade, excitabilidade e sobrevivência neuronal e de células da glia, com receptores nas regiões do cérebro envolvidas na regulação do humor, tais como o córtex pré-frontal, a amígdala, o hipocampo, o corpo estriado e o tálamo. 
Dentre as condições mais fortemente relacionadas às alterações dos hormônios sexuais, encontramos o transtorno disfórico prémenstrual (TDPM), que é caracterizado por uma constelação de sintomas emocionais e alterações comportamentais, que se manifesta com um padrão temporal cíclico em associação com o período pré-menstrual; e, as alterações do humor no climatério, perimenopausa e menopausa. Esta constatação, no entanto, não pressupõe que toda mulher apresente TDPM ou alterações do humor no climatério, perimenopausa e menopausa. Mulheres com estes quadros teriam uma sensibilidade diferenciada às oscilações normais de hormônios, ou seja, não se trata de alterações hormonais, e sim sensibilidade individual diferenciada a tais alterações. ${ }^{6}$

Contudo, a relação hormonal com as condições supracitadas e uma possível relação entre elas, considerando a sensibilidade individual, não estão esclarecidas pela literatura científica. Neste contexto, o presente estudo justifica-se pela necessidade de reunir mais elementos que possam fomentar esta discussão. Além disso, é preciso estimular as equipes das Estratégias de Saúde da Família para o desenvolvimento de ações que contemplem as necessidades da mulher em todos os ciclos de vida e que estejam atentas e intervenham nos fatores de risco para o desenvolvimento da depressão, condição que interfere na qualidade de vida.

Considerando a relevância da temática, este estudo teve por objetivo verificar se existe associação entre o histórico de transtornos disfóricos no período reprodutivo e a presença de sintomas sugestivos de depressão na pósmenopausa em mulheres residentes no interior do Estado do Rio Grande do Sul, Brasil.

\section{MÉTODO}

Este estudo é parte da pesquisa institucional "Estudo Multidimensional de Mulheres Pósmenopausa do Município de Catuípe, RS", da Universidade Regional do Noroeste do Rio
Grande do Sul (UNIJUÍ) e trata-se de um estudo observacional transversal analítico. A pesquisa institucional teve início no ano de 2008 com uma previsão de execução até 2013. Os dados apresentados neste estudo são referentes ao período de março de 2008 a outubro de 2009.

A população da pesquisa institucional foram mulheres com idade entre 50 e 65 anos, em período pós-menopausa, com no mínimo 12 meses de amenorreia e residentes na área urbana e rural do município. Este município, em 2008, contava com 9.582 habitantes, dos quais 4.789 mulheres. Do total de mulheres, 891 encontravam-se na faixa etária do estudo. Considerando $95 \%$ de confiabilidade e uma proporção de $50 \%$, obteve-se um tamanho de amostra de 269. Para a composição da amostra, utilizou-se a técnica de amostragem estratificada proporcional, considerando estratos as microáreas que compõem o território sanitário do município.

A abordagem à população foi possível com o apoio da Secretaria Municipal de Saúde, por meio dos Agentes Comunitários de Saúde que auxiliaram no levantamento do número de mulheres elegíveis para o estudo. Para o presente estudo, foram selecionadas 112 mulheres do banco de dados da pesquisa institucional, 63,3\% da área urbana e $36,6 \%$ da rural que tinham registros completos das variáveis de interesse deste estudo, representando $42 \%$ do tamanho da amostra do estudo maior.

Os dados foram obtidos por meio de entrevista e aplicação de protocolo. A entrevista abordou condições sociodemográficas, aspectos relacionados a terapia de reposição hormonal (TRH) e inquérito sobre presença de transtornos disfóricos. A presença de transtorno disfórico prémenstrual foi obtida por meio de recordatório dos três últimos ciclos menstruais. Para caracterizar transtorno disfórico, esses sintomas deveriam estar presentes na semana antecedente à menstruação e com alívio na semana pós-menstrual. Os sintomas inqueridos foram: tristeza, raiva, irritabilidade, 
nervosismo, confusão, isolamento social, cansaço, mastalgia, distensão abdominal, cefaleia, inchaço em mãos e pernas, aumento de peso e dores articulares ou musculares.

Para avaliar sintomas sugestivos de depressão, utilizou-se a Escala de Hamilton. Trata-se de uma escala validada e já aplicada na população brasileira, estruturada em 21 itens. A resposta da intensidade do sintoma se dá numa escala de 0 a 4 (ausente a intenso). A pontuação final varia de 0 a 62 pontos, sendo considerada depressão leve com escore de 7-17 pontos, moderada entre 1824 pontos e grave maior que 24 pontos.

Para análise dos dados, utilizaram-se a estatística descritiva (média, limite superior, limite inferior, desvio-padrão), teste quiquadrado com uma confiança de $95 \%$, sendo estatisticamente significativos os testes que apresentaram $\mathrm{p} \leq 0,05$. As análises foram executadas com base em Field. ${ }^{8}$

O projeto de pesquisa foi aprovado sob parecer consubstanciado no.124/2009 pelo Comitê de Ética em Pesquisa da Universidade Regional do Noroeste do Estado do Rio Grande do Sul (UNIJUÍ); todas as participantes assinaram o Termo de Consentimento Livre e Esclarecido (TCLE).

\section{RESULTADOS}

Atenderam aos critérios de inclusão no estudo

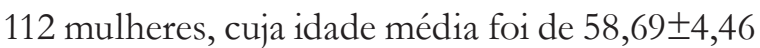
anos, todas entre 50 e 65 anos. Com relação ao perfil sociodemográfico, $75,9 \%$ (85) eram casadas; $69,6 \%$ (78) não concluíram o ensino fundamental; e $60,7 \%$ (68) das mulheres que possuíam renda própria relataram receber de um a dois salários mínimos.

O tempo médio relatado de amenorreia foi de 10,22 $\pm 6,18$ anos. Das mulheres do estudo, segundo relato, $66 \%$ não fizeram uso de terapia de reposição hormonal (TRH) no período do climatério; já 34\% fizeram uso de TRH neste período.

A tabela 1 mostra a distribuição dos transtornos disfóricos de acordo com a intensidade do sintoma relatado, observando-se que todos os sintomas estavam presentes no período pré-menstrual, "às vezes" ou "sempre". Ao observar o percentual da presença dos sintomas, verifica-se que os sentimentos de tristeza, raiva, irritabilidade, nervosismo, cansaço, mastalgia, distensão abdominal, cefaleia e dores articulares e musculares tiveram prevalência superior a $50 \%$, ou seja, mais da metade das mulheres do estudo experimentaram esses sintomas pré-menstruais. Destaca-se ainda que o nervosismo foi o sintoma mais relatado pelas mulheres. 
Tabela 1 - Histórico de transtornos disfóricos pré-menstuais, de acordo com a intensidade do sintoma. Catuípe, RS, 2008-2009.

\begin{tabular}{lcccc}
\hline \multicolumn{1}{c}{ Transtornos disfóricos } & $\begin{array}{c}\text { Nunca } \\
\%(\mathrm{n})\end{array}$ & $\begin{array}{c}\text { Às vezes } \\
\%(\mathrm{n})\end{array}$ & $\begin{array}{c}\text { Sempre } \\
\%(\mathrm{n})\end{array}$ & $\begin{array}{c}\% \text { da presença } \\
\text { do sintoma* }\end{array}$ \\
\hline Tristeza & $43,8(49)$ & $38,4(43)$ & $17,0(19)$ & 55,4 \\
Raiva & $45,5(51)$ & $35,7(40)$ & $17,9(20)$ & 53,6 \\
Irritabilidade & $34,8(39)$ & $43,8(49)$ & $20,5(23)$ & 64,3 \\
Nervosismo & $19,6(22)$ & $55,4(62)$ & $23,2(26)$ & 78,6 \\
Confusão & $63,4(71)$ & $26,8(30)$ & $8,9(10)$ & 35,7 \\
Isolamento social & $67,9(76)$ & $16,1(18)$ & $15,2(17)$ & 31,3 \\
Cansaço & $34,8(39)$ & $37,5(42)$ & $25,0(28)$ & 62,5 \\
Mastalgia & $43,8(49)$ & $33,0(37)$ & $19,6(22)$ & 52,6 \\
Distensão abdominal & $37,5(42)$ & $43,8(49)$ & $17,0(19)$ & 60,8 \\
Cefaleia & $34,8(39)$ & $39,3(44)$ & $24,1(27)$ & 63,4 \\
Inchaço em mãos/pernas & $56,3(63)$ & $26,8(30)$ & $14,3(16)$ & 41,1 \\
Aumento de peso & $50,9(57)$ & $26,8(30)$ & $19,6(22)$ & 46,4 \\
Dores art. e musculares & $35,7(40)$ & $37,5(42)$ & $24,1(27)$ & 61,6 \\
\hline
\end{tabular}

* considerando as respostas "às vezes" e "sempre".

Quanto à presença de sintomas sugestivos de depressão, verificou-se, de acordo com a escala de Hamilton, que 66,1\% (74) das mulheres do estudo apresentaram sintomas depressivos; $53,6 \%$ (60), sintomas depressivos leves; $10,7 \%$ (12), sintomas depressivos moderados; e 1,8\% (2), sintomas depressivos graves.

A aplicação da escala de Hamilton (0 a 62 pontos) apresentou uma média de 9,76 $\pm 5,62$ pontos, um mínimo de um ponto e o máximo 30 pontos. Ao analisar o intervalo de confiança, observou-se que $95 \%$ das mulheres atingiram pontuação na escala de Hamilton entre 8,70 e 10,82 pontos, o que se ajusta com depressão leve.
Ressalta-se que $100 \%$ das mulheres com sintomas sugestivos de depressão relataram a presença de um ou mais transtornos disfóricos pré-menstruais. A tabela 2 mostra a frequência da presença e intensidade de transtornos disfóricos segundo o nível de depressão. Ao analisar o percentual da presença de sintomas entre as mulheres com quadro depressivo, observa-se que estas experienciaram os sintomas disfóricos "às vezes" ou "sempre" nos ciclos menstruais. Esses dados revelam que a prevalência de transtornos disfóricos é elevada entre as mulheres que apresentam sintomas sugestivos de depressão no período pós-menopausa. 
Tabela 2 - Frequência e intensidade de transtornos disfóricos, segundo o nível de depressão. Catuípe, RS, 2008-2009.

\begin{tabular}{|c|c|c|c|c|c|}
\hline \multicolumn{2}{|c|}{ Transtornos disfóricos } & $\begin{array}{c}\text { SDL } \\
\% \\
42,9\end{array}$ & $\begin{array}{c}\text { SDM } \\
\% \\
4,1\end{array}$ & $\begin{array}{c}\text { SDG } \\
\% \\
-\end{array}$ & $\begin{array}{c}\begin{array}{c}\% \text { da presença } \\
\text { do sintoma** }\end{array} \\
47\end{array}$ \\
\hline Tristeza & $\begin{array}{l}\text { Nunca } \\
\text { Às vezes } \\
\text { Sempre }\end{array}$ & $\begin{array}{l}42,9 \\
58,1 \\
68,4\end{array}$ & $\begin{array}{c}4,1 \\
14,0 \\
21,1\end{array}$ & $\begin{array}{c}- \\
5,3 \\
5,3\end{array}$ & $\begin{array}{c}47 \\
77,4 \\
94,8\end{array}$ \\
\hline Raiva & $\begin{array}{l}\text { Nunca } \\
\text { Às vezes } \\
\text { Sempre }\end{array}$ & $\begin{array}{l}43,1 \\
50,0 \\
85,0\end{array}$ & $\begin{array}{c}3,9 \\
20,0 \\
10,0\end{array}$ & $\begin{array}{c}- \\
2,5 \\
5,0\end{array}$ & $\begin{array}{c}47 \\
72,5 \\
100\end{array}$ \\
\hline Irritabilidade & $\begin{array}{l}\text { Nunca } \\
\text { Às vezes } \\
\text { Sempre }\end{array}$ & $\begin{array}{l}48,7 \\
44,9 \\
78,3\end{array}$ & $\begin{array}{c}5,1 \\
14,3 \\
13,0\end{array}$ & $\begin{array}{c}- \\
2,0 \\
4,3\end{array}$ & $\begin{array}{l}53,8 \\
61,2 \\
95,6\end{array}$ \\
\hline Nervosismo & $\begin{array}{l}\text { Nunca } \\
\text { Às vezes } \\
\text { Sempre }\end{array}$ & $\begin{array}{l}45,5 \\
50,0 \\
65,4\end{array}$ & $\begin{array}{c}4,5 \\
11,3 \\
15,4\end{array}$ & $\begin{array}{c}4,5 \\
- \\
3,8\end{array}$ & $\begin{array}{l}54,5 \\
61,3 \\
84,6\end{array}$ \\
\hline Confusão & $\begin{array}{l}\text { Nunca } \\
\text { Às vezes } \\
\text { Sempre }\end{array}$ & $\begin{array}{l}52,1 \\
50,0 \\
70,0\end{array}$ & $\begin{array}{c}5,6 \\
20,0 \\
20,0\end{array}$ & $\begin{array}{c}- \\
6,7 \\
-\end{array}$ & $\begin{array}{c}57,7 \\
76,7 \\
90\end{array}$ \\
\hline Isolamento social & $\begin{array}{l}\text { Nunca } \\
\text { Às vezes } \\
\text { Sempre }\end{array}$ & $\begin{array}{l}52,6 \\
50,0 \\
58,8\end{array}$ & $\begin{array}{l}6,6 \\
11,1 \\
29,4\end{array}$ & $\begin{array}{l}- \\
5,6 \\
5,9\end{array}$ & $\begin{array}{l}59,2 \\
66,7 \\
94,1\end{array}$ \\
\hline Cansaço & $\begin{array}{l}\text { Nunca } \\
\text { Às vezes } \\
\text { Sempre }\end{array}$ & $\begin{array}{l}38,5 \\
57,1 \\
67,9\end{array}$ & $\begin{array}{l}10,3 \\
11,9 \\
10,7\end{array}$ & $\begin{array}{c}2,6 \\
- \\
3,6\end{array}$ & $\begin{array}{c}51,4 \\
69 \\
82,2\end{array}$ \\
\hline Mastalgia & $\begin{array}{l}\text { Nunca } \\
\text { Às vezes } \\
\text { Sempre }\end{array}$ & $\begin{array}{l}55,1 \\
37,8 \\
68,2\end{array}$ & $\begin{array}{c}10,2 \\
8,1 \\
18,2\end{array}$ & $\begin{array}{c}- \\
2,7 \\
4,5\end{array}$ & $\begin{array}{l}65,3 \\
48,6 \\
90,9\end{array}$ \\
\hline Distenção abdominal & $\begin{array}{l}\text { Nunca } \\
\text { Às vezes } \\
\text { Sempre }\end{array}$ & $\begin{array}{l}50,0 \\
51,0 \\
63,2\end{array}$ & $\begin{array}{c}9,5 \\
10,2 \\
15,8\end{array}$ & $\begin{array}{c}2,4 \\
- \\
5,3\end{array}$ & $\begin{array}{l}61,9 \\
61,2 \\
84,3\end{array}$ \\
\hline Cefaleia & $\begin{array}{l}\text { Nunca } \\
\text { Às vezes } \\
\text { Sempre }\end{array}$ & $\begin{array}{l}43,6 \\
59,1 \\
55,6\end{array}$ & $\begin{array}{c}7,7 \\
6,8 \\
22,2\end{array}$ & $\begin{array}{c}- \\
2,3 \\
3,7\end{array}$ & $\begin{array}{l}51,3 \\
68,2 \\
81,5\end{array}$ \\
\hline Inchaço em mãos/pernas & $\begin{array}{l}\text { Nunca } \\
\text { Às vezes } \\
\text { Sempre }\end{array}$ & $\begin{array}{l}52,4 \\
50,0 \\
56,3\end{array}$ & $\begin{array}{c}7,9 \\
10,0 \\
25,0\end{array}$ & $\begin{array}{c}1,6 \\
3,3 \\
-\end{array}$ & $\begin{array}{l}61,9 \\
63,3 \\
81,3\end{array}$ \\
\hline Aumento de peso & $\begin{array}{l}\text { Nunca } \\
\text { Às vezes } \\
\text { Sempre }\end{array}$ & $\begin{array}{l}56,1 \\
46,7 \\
50,0\end{array}$ & $\begin{array}{c}7,0 \\
10,0 \\
22,7\end{array}$ & $\begin{array}{c}1,8 \\
- \\
4,5\end{array}$ & $\begin{array}{l}64,9 \\
56,7 \\
77,2\end{array}$ \\
\hline $\begin{array}{l}\text { Dores articulares ou } \\
\text { musculares }\end{array}$ & $\begin{array}{l}\text { Nunca } \\
\text { Às vezes } \\
\text { Sempre }\end{array}$ & $\begin{array}{l}40,0 \\
66,7 \\
48,1\end{array}$ & $\begin{array}{c}10,0 \\
4,8 \\
22,2\end{array}$ & $\begin{array}{c}2,5 \\
- \\
3,7\end{array}$ & $\begin{array}{c}52,5 \\
71,5 \\
74\end{array}$ \\
\hline
\end{tabular}

**Percentual da presença de sintomas em mulheres com sintomas depressivos. $\mathrm{SDL}=$ sintomas depressivos leves; $\mathrm{SDM}=$ sintomas depressivos moderados; $\mathrm{SDG}=$ sintomas depressivos graves. 
Ao se aplicar teste estatístico para identificar se há diferença entre o grupo de mulheres que apresentou sintomas depressivos e o que não apresentou, verificou-se associação estatisticamente significativa entre depressão e os seguintes sintomas: tristeza, raiva, irritabilidade, confusão, isolamento social, cansaço, cefaleia e dores articulares ou musculares. A tabela 3 apresenta a distribuição dos sintomas de transtornos disfóricos pré-menstruais, segundo a presença de depressão.

Tabela 3 - Distribuição dos sintomas de transtornos disfóricos pré-menstruais, segundo a presença de depressão. Catuípe, RS, 2008-2009.

\begin{tabular}{lccc}
\hline \multicolumn{1}{c}{ Transtornos disfóricos } & $\begin{array}{c}\text { Presença de sintomas depressivos } \\
\%(\mathrm{n})\end{array}$ & $\begin{array}{c}\text { Sem sintomas depressivos } \\
\%(\mathrm{n})\end{array}$ & $\mathrm{p}$ \\
\hline Tristeza & $80,0(50)$ & $19,4(12)$ & $0,01^{*}$ \\
Raiva & $82,8(64)$ & $17,2(11)$ & $0,01^{*}$ \\
Irritabilidade & $72,2(52)$ & $27,8(20)$ & $0,05^{*}$ \\
Nervosismo & $68,5(61)$ & $31,5(28)$ & 0,21 \\
Confusão & $80,0(32)$ & $20,0(8)$ & $0,01^{*}$ \\
Isolamento social & $80,0(28)$ & $20,0(7)$ & $0,03^{*}$ \\
Cansaço & $74,3(52)$ & $25,7(18)$ & $0,01^{*}$ \\
Mastalgia & $64,4(38)$ & $35,6(21)$ & 0,86 \\
Distensão abdominal & $67,6(46)$ & $32,4(22)$ & 0,53 \\
Cefaleia & $73,2(52)$ & $26,8(19)$ & $0,02^{*}$ \\
Aumento de peso & $65,4(34)$ & $34,6(18)$ & 0,95 \\
Dores articulares ou & $72,5(50)$ & $27,5(19)$ & $0,03^{*}$ \\
musculares & & & \\
\hline
\end{tabular}

* Estatisticamente significativo $(\mathrm{p} \leq 0,05)$.

Outra análise realizada foi com relação ao uso de terapia de reposição hormonal quanto à presença ou não de sintomas depressivos. Das mulheres que realizaram TRH, 67\% tiveram sintomas de depressão, e das que não realizaram, $74 \%$ tiveram sintomas de depressão. Das que fizeram uso de TRH até dois anos, 36,0\%(9) não apresentaram sintomas depressivos; 48,0\%(12) apresentaram sintomas depressivos leves; 12,0\%(3), sintomas depressivos moderados; e $4,0 \%(1)$, sintomas depressivos graves. Das que fizeram uso de dois a quatro anos $25,0 \%$ (1) não apresentaram sintomas depressivos, 50,0\%(2) apresentaram sintomas depressivos leves e 25,0\%(1) apresentaram sintomas depressivos moderados. Das que relataram terem usado TRH por mais de quatro anos, $44,4 \%(4)$ não apresentaram sintomas depressivos e 55,5\%(5), sintomas depressivos leves. Não se verificou, contudo, relação entre o uso de reposição de terapia hormonal e a presença de sintomas sugestivos de depressão $(p=0,81)$.

\section{DISCUSSÃO}

Este estudo teve por objetivo verificar se existe associação entre o histórico de transtornos disfóricos no período reprodutivo e a presença de sintomas sugestivos de 
depressão na pós-menopausa em mulheres residentes no interior do Estado do Rio Grande do Sul, Brasil. Na população, a prevalência de depressão foi de $66,1 \%$.

No Brasil há poucos estudos em regiões distantes de grandes centros; da mesma forma, há poucos estudos voltados a mulheres que vivenciam o período pós-menopausa. A importância de estudos com esta população está em identificar variáveis que possam interferir de forma negativa na qualidade de vida e, desta forma, propor alternativas para melhor viver esta fase da vida e na velhice. No século passado, grande parte delas não alcançava a menopausa; hoje a expectativa é que as mulheres vivam um terço de suas vidas após a menopausa. ${ }^{9}$

A menopausa é dividida em duas etapas principais: a perimenopausa, que se caracteriza pela presença de ciclos irregulares ou com características diferentes dos ciclos observados durante a vida reprodutiva, tendo a última menstruação, ocorrido há menos de 12 meses; e a pós-menopausa, pela ausência de menstruação por mais de 12 meses.

A maioria das mulheres que atinge a menopausa não apresenta sintomas depressivos proeminentes; no entanto, uma prevalência maior do que a esperada de sintomas depressivosímiles vem sendo observada entre elas. ${ }^{9}$ Hay \& Johnstone, ${ }^{10}$ contudo, destacam que a transição para a menopausa parece agir como facilitadora e não como causadora dos sintomas do humor.

Entre as discussões acerca da relação entre depressão e menopausa, e suas causas, a pesquisa de Dennerstein ${ }^{11}$ colabora no sentido de colocar em discussão se a depressão é gerada por alterações biológicas ou por causas psicossociais. Seu estudo aponta que a maior tendência a depressão no climatério tem sido atribuída ao medo de envelhecer e a percepção de proximidade da morte, sentimentos estes agravados pela sensação de inutilidade ou carência afetiva. O climatério é uma fase de transição física e social, coincidindo com a independência dos filhos, a morte de familiares e a aposentadoria, circunstâncias estas que requerem ajustes emocionais difíceis para a mulher.

Já o estudo de Lorenzi $^{12}$ discute sobre vantagens e desvantagens sobre a menopausa. Em seus achados, verificou que a percepção da menopausa como evento desvantajoso (atitude negativa) associou-se a pior sintomatologia climatérica, reforçando que atitudes negativas repercutem na manifestação de piores sintomas. Favarato $^{13}$ destaca que outro aspecto que colabora para a depressão é a imagem que a mulher constrói de si mesma nesse período, decorrente da sua baixa-estima acarretada pelo humor depressivo, pelo sentimento de estar envelhecendo fisicamente e a tendência ao aumento de peso corporal, fatos que contribuem para uma autoimagem negativa que as levam a se sentir menos atraentes e desejadas.

Alterações do humor são queixas relatadas com grande frequência aos especialistas de clínicas de menopausa, mas para Fernandes et al., ${ }^{14}$ outros estressores, característicos desse período da vida, podem contribuir para os quadros depressivos climatéricos, e não apenas a senescência ou o declínio hormonal. Estudos comunitários apontam que a sintomatologia depressiva em mulheres na meia-idade está associada a fatores como atitude em relação à menopausa, saúde geral da mulher, ansiedade, problemas de relacionamento, presença de outros sintomas climatéricos e histórico de episódio depressivo anterior.

No estudo de Lorenzi ${ }^{15}$ sobre a qualidade de vida, cerca de 50 a $70 \%$ das mulheres vieram a referir sintomas somáticos e dificuldades emocionais no climatério, interferindo no seu bem-estar. São também comuns queixas relacionadas a dificuldades como sono, perda da libido, cefaleia, mialgias e artralgias, maior ansiedade e até depressão. Ressalta-se que, assim como no estudo de Lorenzi, também no presente estudo se encontrou a maioria dessas queixas. Persistem, no entanto, controvérsias sobre o quanto tais queixas são decorrentes 
somente da queda estrogênica ou de fatores psicossociais relacionados ao próprio processo de envelhecimento. As ondas de calor e a atrofia urogenital seriam as únicas queixas diretamente relacionadas à queda dos níveis estrogênicos. As demais estariam relacionadas sobretudo à dificuldade de aceitar o processo de envelhecimento, este simbolizado pela menopausa..$^{15}$

Em relação ao histórico de transtornos disfóricos na população do presente estudo, verificou-se que todos os sintomas estavam presentes "às vezes" ou "sempre" no período prémenstrual, considerando o recordatório dos três últimos ciclos. Destes, os que foram relatados com maior frequência foram: o sentimento de tristeza, raiva, irritabilidade, nervosismo, cansaço, mastalgia, distensão abdominal, cefaleia e dores articulares e musculares. Achados do presente estudo revelam associação estatisticamente significativa entre depressão e os seguintes sintomas: tristeza, raiva, irritabilidade, confusão, isolamento social, cansaço, cefaleia e dores articulares ou musculares.

Neste estudo, observou-se associação entre transtornos disfóricos pré-menstruais e sintomatologia sugestiva de depressão; e ainda, as mulheres que apresentaram sintomas de depressão considerados graves relataram ter sentido "às vezes" ou "sempre" todos os sintomas disfóricos, sugerindo que a sintomatologia que antecede a menopausa pode estar relacionada com a susceptibilidade à depressão. Este resultado refuta os achados de outros estudos de NicolSmith ${ }^{16}$ e Petticrew: ${ }^{17}$ ambos não encontraram relação entre menopausa e depressão.

Segundo Stewart \& Boydell, ${ }^{18}$ mulheres que tiveram histórico de transtorno depressivo ansioso ao longo da vida têm maior probabilidade de apresentarem outro episódio, enquanto outras podem sentir pela primeira vez insônia, irritabilidade, alterações de humor, ausência de desejo sexual e fadiga na menopausa. E ainda, segundo pesquisa conduzida por Pedro et al., ${ }^{19}$ os sintomas emocionais são as queixas mais frequentes entre as mulheres brasileiras que procuram atendimento ginecológico.

Neste estudo, verificou-se associação entre TDPM e depressão, achado que corrobora a literatura científica, uma vez que há poucos e discutíveis estudos sobre o tema. Contudo, trabalhos importantes e relacionados ao tema, como o de Cunningham et al., ${ }^{20}$ afirmam que mulheres com TDPM relatam maior prevalência de transtornos psiquiátricos, especialmente do humor, com valores entre $30 \%$ e $70 \%$, predizendo maior risco para futuros episódios de depressão ao longo da vida, em especial relacionado ao ciclo reprodutivo feminino como a perimenopausa.

Estudo de Silva et al., ${ }^{21}$ que teve como objetivo avaliar a prevalência de depressão em mulheres climatéricas, observou presença de TDPM em $44,3 \%$ do total da população do estudo, o mesmo percentual que apresentou episódios depressivos prévios. Verificou, ainda, associação entre depressão e menopausa, ressaltando, no entanto, que sobre o TDPM existem escassos trabalhos que subsidiem este achado, assunto considerado discutível.

Das limitações do presente estudo, destaca-se que por se tratar de estudo transversal dados sobre transtornos disfóricos, obtidos por recordatório dos últimos três ciclos, podem não expressar o ocorrido por toda a fase reprodutiva. Os estudos realizados sobre este tema são bastante controversos e demandam mais elementos que possam corroborar a discussão.

\section{CONCLUSÃO}

Concluiu-se que existe associação de transtornos disfóricos pré-menstruais e sintomas sugestivos de depressão em mulheres pósmenopausa. A temática apresentada neste estudo não se esgota e requer mais investigações para que se possa comparar achados para melhor compreensão da relação da menopausa com sintomas depressivos. Por ora, a contribuição deste trabalho fica centrado na associação dos 
sintomas de transtornos disfóricos com sintomas sugestivos de depressão em mulheres pósmenopausa residentes no interior do Rio Grande do Sul, Brasil.

Os resultados apresentados abrem a discussão sobre a importância de implementar políticas de atenção voltadas para o cuidado da mulher nessa etapa de vida e realizar mais investigações com mulheres com perfil semelhante à população do estudo subsidiando esses achados. Os indícios de sintomas sugestivos de depressão nesta população são expressivos, e muitos fatores podem estar associados a esta condição. O acompanhamento pelas equipes de saúde da Estratégia da

\section{REFERÊNCIAS}

1. De Lorenzi DRS, Baracat EC, Saciloto B, Padilha Júnior I. Fatores associados à qualidade de vida após menopausa. Rev Assoc Med Bras 2006;52(5):312-7.

2. Zahar SE, Aldrighi JM, Pinto Neto AM, Conde DM, Zahar LO, Russomano F. Qualidade de vida em usuárias e não usuárias de terapia de reposição hormonal. Rev Assoc Med Bras 2005;51(3):133-8.

3. Vieira CS, Navarro PAA. Como diagnosticar e tratar: síndrome climatérica. Rev Bras Med 2007;64:99-109.

4. Nievas AF, Furegato ARF, Iannetta O, Santos JLF. Depressão no climatério: indicadores biopsicossociais. J Bras Psiquiatr 2006;55(4):274-9.

5. Del Porto JA. Depressão: conceito e diagnóstico. Rev Bras Psiquiatr 1999;21:6-11.

6. Rubinow DR, Schimidt PJ. Gonadal Steroid regulation of mood: the lessons of premenstrual syndrome. Front Neuroendocrinol 2006;27(2):210-6.

7. Hamilton M. A rating scale for depression. J Neurol Neurosurg Psychiatry 1960;23:56-62.

8. Field A. Descobrindo a estatística usando Spss. 2. ed. Porto Alegre: Artmed; 2009.

9. Maartens LW, Knottnerus JA, Pop VJ. Menopausal transition and increased depressive symptomatology: a community based prospective study. Maturitas 2002;42(3):195-200.
Família, contudo, é de grande relevância, tanto informando e educando sobre as mudanças que ocorrem no corpo da mulher com a chegada da menopausa, como intervindo precocemente em situações especiais. A pós-menopausa não deve ser sentida pela mulher como uma fase deletéria, mas uma fase de plenitude.

\section{AGRADECIMENTOS}

Agradecemos à Universidade Regional do Noroeste do Estado do Rio Grande do Sul (UNIJUÍ), pelo financiamento de horas de pesquisa, e à Prefeitura Municipal de CatuípeRS, pelo apoio à viabilização desta pesquisa.
10. Hay AG, Bancroft J, Johnstone EC. Affective symptoms in women attending a menopause clinic. Br J Psychiatry 1994:164(4):513-6.

11. Dennerstein L, Lehert P, Guthrie J. The effects of the menopausal transition and biopsychosocial factors on well-being. Arch Womens Ment Health 2002;5(1):15-22.

12. Lorenzi DRS, Danelon C, Saciloto B, Padilha Jr I. Fatores indicadores da sintomatologia climatérica. Rev Brasil Ginecol Obstet [Internet]. 2005 [acesso em XX dez 20XX];27(1):12-9. Disponível em: http:// www.scielo.br/pdf/rbgo/v27n1/24286.pdf.

13. Favarato MECS, Aldrighi JM, Fráguas Junior R, Pires ALR, Lima SMRR. Sexualidade e climatério: influência de fatores biológicos, psicológicos e sócio-culturais. Reprod Clim [Internet]. 2000 [acesso em XX dez 20XX];15(4):199-202. Disponível em: http://bases.bireme.br/cgibin/wxislind.exe/iah/ online/?IsisScript $=$ iah $/$ iah. $x i s \& s r c=$ google\&base $=$ LI LACS\&lang $=$ p\&next Action $=\operatorname{lnk} \&$ exprSearch $=28912$ 5\&indexSearch=ID.

14. Fernandes RCL, Rozenthal M. Avaliação da sintomatologia depressiva em mulheres de mulheres no climatério com a escala de rastreamento populacional para depressão CES-D. Rev Psiquiatr Rio Gd Sul [Internet]. 2008 [acesso em XX dez 20XX];30(3):192-200. Disponível em: http://www. scielo.br/pdf/rprs/v30n3/v30n3a08.pdf 
15. De Lorenzi DR. Avaliacao da qualidade de vida no climaterio. Rev Bras Ginecol Obstet 2008;30(3):103-6.

16. Nicol-Smith L. Causality, menopause, and depression: a critical review of the literature. BMJ 1996;313(7067):1229-32.

17. Petticrew M. Causality, the menopause, and depression. Review did not fully examine the evidence. BMJ 1997;314(7080):608.

18. Stewart DE, Boydell KM. Psychologic distress during menopause: associations across the reproductive life cycle. Int J Psychiatry Med 1993;23(2):157-62.
19. Pedro AO, Pinto-Neto AM, Costa-Paiva L, Osis MJ, Hardy E.Procura de serviço médico por mulheres climatéricas brasileiras. Rev Saúde Pública 2002;36(4):484-90.

20. Cunningham J, Yonkers KA, O’Brien S, Eriksson E. Update on research and treatment of premenstrual dysphoric disorder. Harv Rev Psychiatry 2009; 17(2):120-37.

21. Silva MNM, Brito LMO, Chein MBC, Brito LGO, Navarro PAAS. Depressão em mulheres climatéricas: análise de mulheres atendidas ambulatorialmente em um hospital universitário no Maranhão. Rev Psiquiatr Rio Gd Sul 2008;30(2):150-4. 